\title{
Enrichment of wearable sensor data from individuals with lower limb amputation in a free-living setting
}

\author{
Alexander Jamieson ${ }^{1}$, Laura Murray ${ }^{1}$, Arjan Buis $^{1}$ \\ ${ }^{1}$ University of Strathclyde, Glasgow UK

\section{Background}

Objective physical activity monitoring of lower limb amputees can be achieved using wearable sensors, however the literature has shown that simplistic objective monitoring (for instance, measuring step count or energy expenditure) is overwhelmingly favoured (Jamieson et al., 2020). For a more detailed analysis of activity, the implementation of machine learning algorithms to recognize wider varieties of activity is a logical solution. Currently, no research has attempted to perform HAR with an amputee population using an unsupervised learning approach.

\section{Aim}

To provide clinically useful information for healthcare professionals specialising in lower limb amputee rehabilitation by developing a robust unsupervised classification system that can recognize different activities and walking terrains covered by lower limb amputees.

\section{Method}

The following methodology was given ethical approval by the University of Strathclyde's Ethics Committee. A combination of amputee volunteers with no known comorbidities and healthy volunteers with no known gait impairments were recruited for the study. The participants were instructed to utilize an IMU and to record themselves with a chest-mounted camera while going on a walk on a variety of terrains in the local vicinity of their homes. The collected IMU data was used to train an unsupervised classification system to recognize walking activities and terrains, with the annotated data from the camera providing validation to the performance of the classifier.

\section{Results}

12 participants were recruited for the study. This demographic is comprised of 8 healthy individuals, 3 transtibial amputees and 1 bilaterial amputee. The development of the classification algorithm is an ongoing process. Principal component analysis is applied to reduce the dimensionality of the feature set to 2 dimensions. The K-Means clustering algorithm assigns 20 cluster groups, with the intention that each cluster should be equivalent to a different type of activity or terrain. The resultant clustering model can be seen in figure 1. Mathematically, the equivalency of the cluster and class labels is calculated via normalized mutual information, which is currently at $31.1 \%$. 


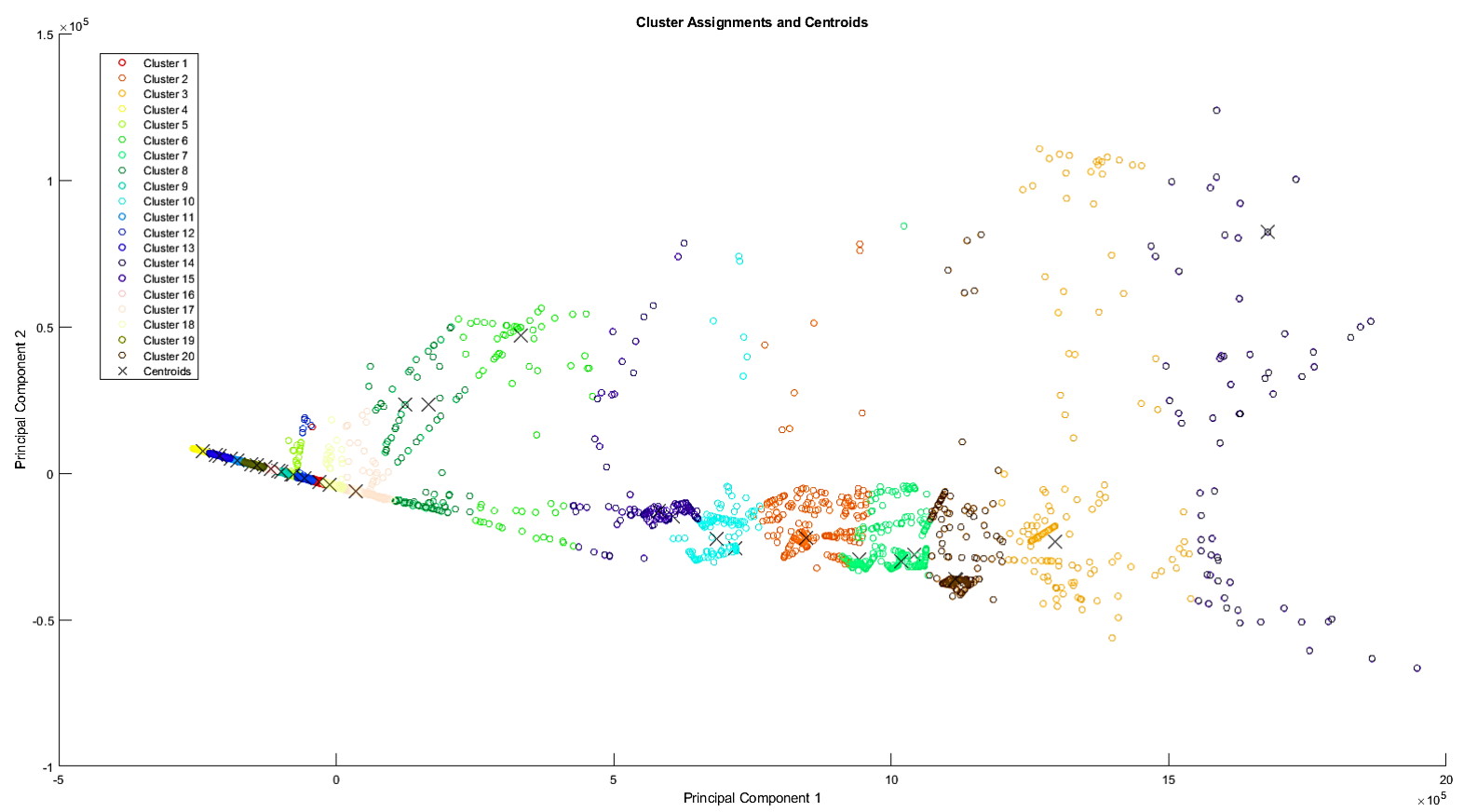

Figure 1 - Clusters of the dataset $(n=20)$ in the Principal Component space, attained via K-Means Clustering

\section{Discussion}

$99 \%$ of the variability in the dataset is explained by the first principal component, which is skewed towards the data from the bilateral amputee, suggesting a separate clustering model should be constructed.

Additionally, similarity between labels will be analysed to determine the trade-off in the hierarchical detail in the labels and the cluster model performance.

While optimisation of the classifier is still ongoing, the clustering model shows potential to recognize different types of walking terrains for lower limb amputees.

\section{References}

Jamieson, A., Murray, L. \& Buis, A. 2020. The Use Of Physical Activity Outcomes In Rehabilitation Interventions For Lower Limb Amputees: A Systematic Review. Canadian Prosthetics \& Orthotics Journal, 3.

\section{Acknowledgements}

This research was developed for Alexander Jamieson's PhD thesis. Contribution to student fees are provided by PAL Technologies Ltd. 